\title{
Online action planning forums to develop a roadmap to mitigate the impact of COVID-19 on the delivery of global children's surgical \\ care
}

\author{
Alizeh Abbas ${ }^{1}$ (1) - Lubna Samad ${ }^{1} \cdot$ Doruk Ozgediz $^{2}$ - Adesoji Ademuyiwa ${ }^{3} \cdot$ Emmanuel A. Ameh $^{4} \cdot$ Tahmina Banu $^{5}$. \\ Fabio Botelho ${ }^{6} \cdot$ Beda Espineda $^{7} \cdot$ Zipporah Gathuya $^{8} \cdot$ Kokila Lakhoo $^{9} \cdot$ Lawal-Aiyedun Olubunmi $^{10}$. \\ Vrisha Madhuri $^{11} \cdot$ Leecarlo Millano $^{12} \cdot$ Susane Nabulindo ${ }^{13} \cdot$ Sameh Shehata $^{14} \cdot$ Kenneth Wong $^{15}$. \\ Marilyn W. Butler ${ }^{16}$. On behalf of the Global Initiative for Children's Surgery
}

Accepted: 29 March 2021 / Published online: 21 April 2021

(c) The Author(s), under exclusive licence to Springer-Verlag GmbH Germany, part of Springer Nature 2021

\begin{abstract}
Purpose We aimed to understand the challenges facing children's surgical care providers globally and realistic interventions to mitigate the catastrophic impact of COVID-19 on children's surgery.

Methods Two online Action Planning Forums (APFs) were organized by the Global Initiative for Children's Surgery (GICS) with a geographically diverse panel representing four children's surgical, anesthesia, and nursing subspecialties. Qualitative analysis was performed to identify codes, themes, and subthemes.

Results The most frequently reported challenges were delayed access to care for children; fear among the public and patients; unavailability of appropriate personal protective equipment (PPE); diversion of resources toward COVID-19 care; and interruption in student and trainee hands-on education. To address these challenges, panelists recommended human resource and funding support to minimize backlog; setting up international, multi-center studies for systematic data collection specifically for children; providing online educational opportunities for trainees and students in the form of large and small group discussions; developing best practice guidelines; and, most importantly, adapting solutions to local needs.

Conclusion Identification of key challenges and interventions to mitigate the impact of the COVID-19 pandemic on global children's surgery via an objective, targeted needs assessment serves as an essential first step. Key interventions in these areas are underway.
\end{abstract}

Keywords Global surgery · Children's surgery · Pediatric surgery · COVID-19 · Pandemic · Online social networking

\section{Introduction}

The Severe Acute Respiratory Syndrome Coronavirus 2 (SARS-CoV-2) that led to the global COVID-19 pandemic began as early as December 2019 in Wuhan, China and rapidly spread throughout the world, affecting 10.5 million people and causing 500,000 deaths by 2 July 2020 [1]. According to currently available data, a small proportion of COVID-19 cases have been seen in children [2] and

Alizeh Abbas

alizehabbas.raza@gmail.com

Marilyn W. Butler mwbutler@gmail.com

Extended author information available on the last page of the article adolescents with milder forms of the disease [3-6]. Other children have been affected by a more severe condition called Multisystem Inflammatory Syndrome in Children (MIS-C) [7, 8], with reports of a Kawasaki-like manifestation $[9,10]$. While the direct impact of the pandemic in the younger age group seems to be reassuringly small, it is the indirect consequences of compromised care that are of the greatest concern; reports documenting the significant impact on routine immunization, delays in childhood cancer treatment, and deferred surgical care indicate the extent of the problem [11].

As the pandemic continues to drastically alter health systems and associated prioritization and allocation of resources globally, children's surgery may be overlooked or temporarily forgotten if prompt action is not taken [12]. Mazingi and colleagues identified the possible implications 
of the pandemic on children's surgery in Africa. They made recommendations spanning across seven domains, including surgical rationing and provision, healthcare worker welfare, child protection, and training [13]. They also reiterated that children have unique needs and may be disproportionately affected during stressful times [13]. In addition, a study published in May 2020 in The Lancet by Roberton and colleagues estimated that disruption in essential child health interventions could result in more than 6,000 deaths per day over the next 6 months in low- and middle-income countries (LMICs) [11]. In this context, the Global Initiative for Children's Surgery (GICS)[14] anticipated a catastrophic effect on children's surgical care delivery caused by the diversion of resources to COVID-19-driven public health and clinical priorities, particularly in LMICs with already limited resources. GICS galvanized its existing network in LMICs and High-income Countries (HICs). Treatment guidelines and resources were published on the GICS website as the COVID-19 pandemic was evolving, allowing updated information to reach children's surgical care providers in a timely manner. This included a wide range of relevant topics, including the epidemiology, diagnosis, and treatment of COVID-19 in children, particularly in the surgical patient; impact on surgical care and infrastructure requirements; anesthesia and critical care challenges; and safety precautions for healthcare providers during the pandemic. Most importantly, all these guidelines were adapted to LMIC needs, maximizing their utility for a broad range of resource-limited settings.

GICS leveraged its worldwide network to understand better the challenges faced in different countries by organizing two online Action Planning Forums (APFs). In addition to recognition of situation-specific problems, these forums attempted to identify potential interventions or resources that could be rapidly implemented or funded to mitigate the impact of COVID-19 on children's surgical care. Attended by a large number of participants from across the globe, the APFs provided a unique opportunity to share experiences and expertise with relevant stakeholders. This paper summarizes the discussions of the AFPs, highlighting the challenges faced by children's surgical care providers and the impact of the pandemic on children's surgery, as well as the proposed actions to effectively address these challenges.

\section{Methods}

Panelists for the GICS APFs were chosen across a spectrum of pediatric subspecialties (general surgery, orthopedic surgery, nursing, and anesthesia) representing diverse regions (East Asia, Southeast Asia, South Asia, the Middle East, Sub-Saharan Africa, and South America). After an informal discussion, questions were emailed to the panelists, and panelists consented to video and audio recording of the sessions. Pre-registration at no cost was required for all participants, and a Zoom platform sponsored by Lifebox was employed [15]. Recognizing a sense of "webinar fatigue", the sessions were dubbed "Action Planning Forums" to emphasize their participative and result-oriented mandate and the opportunity to connect providers, policy makers, and donors. The focus was on practical interventions that could be implemented to assist colleagues, particularly in LMICs, allowing GICS and potential donors to get a hands-on understanding of where to invest their resources. The APFs were moderated by the current and incoming GICS Chairs.

The first APF was held on June 4, 2020, and panelists answered five questions (Table 1) about the impact, challenges, and strategies in relation to the pandemic, as well as the response required by GICS. Prepared slides were projected during their presentations. This 90-min session identified gaps in knowledge and on-ground challenges. Information gleaned from the first session helped structure the second session that was held on July 2, 2020, which focused attention on specific actions that GICS and others could take to address the identified gaps. The question for all panelists of the second session included: How can GICS help LMIC colleagues, specifically with educational resources, guidelines, backlog of patients, and research? Panelists also answered participant questions posted on the chat box during the session. Both APFs were held from 1400 to 1530 Greenwich Meridian Time to facilitate participation from multiple time zones.

Notes and transcriptions from the proceedings were uploaded to the qualitative data analysis software MAXQDA 2018. Codes, categories, themes, and subthemes were identified using an inductive coding approach. To minimize any potential bias, the moderators and panelists were not involved in the first round of coding. Several authors met regularly to discuss and review codes, and coding was
Table 1 List of questions asked at the first Action Planning Forum

\footnotetext{
What is the current and projected impact of the pandemic in various regions of the world?

What are local strategies and challenges?

What can the Global Initiative for Children's Surgery and others do to help with disruptions to essential surgical services for children unable to access care?

What are the best roles for telemedicine for education and outreach?

What is needed to help protect health care workers?
} 
continued until thematic saturation was reached. Identified themes were categorized according to the framework domains defined in the Optimal Resources for Children's Surgical Care (OReCS) document [16]. Final results were reviewed by all of the panelists and moderators.

This study received institutional review board (IRB) exemption from the Interactive Research and Development (IRD) IRB at the Indus Hospital, Karachi, Pakistan (IRB number: IRD_IRB_2020_12_005).

\section{Results}

The two APFs had 11 and 8 panelists, respectively (3 panelists were infected with COVID-19 after the first APF and were unable to attend the second APF). There were 364 participants from 47 countries at the first forum (Fig. 1) with an additional 174 YouTube views; the second forum had 157 participants from 34 countries with 7 additional YouTube viewings. The APFs saw a strong participation from LMICs (APF 1: $n=287,78.8 \%$ and APF $2: n=107$, $68.2 \%$ ). The challenges, impact, and the proposed actions brought forth by the panelists align with multiple framework domains identified within the OReCS: human resources and training; infrastructure; service delivery; equipment and supplies; quality improvement; advocacy and awareness; and governance and financing. The APFs also saw participation from leading charity and philanthropic organizations, who used the forum to help fuel their advocacy messages by hearing directly from frontline providers.

\section{Challenges and impact}

When panelists shared their thoughts on the challenges facing them in the COVID-19 era, several common themes emerged. The most frequently reported challenges (Table 2) included cancelation of elective surgery; delayed access to care; fear among patients and the general public; unavailability of resources, especially personal protective equipment (PPE), leading to safety concerns among healthcare workers; diversion of resources away from children's surgery and toward COVID-19 care; and interruption in hands-on clinical training for fellows, residents, and students. Additional challenges and panelists' observations on the impact of the pandemic are listed in Tables 3 and 4.

\section{Proposed actions}

During the second APF, when asked about the role of GICS in helping with educational resources, guidelines, back$\log$, and research, panelists proposed several key actions to
Fig. 1 Number of participants from HICs and LMICs at the GICS In-person conference 2020 and the Online Action Planning Forums

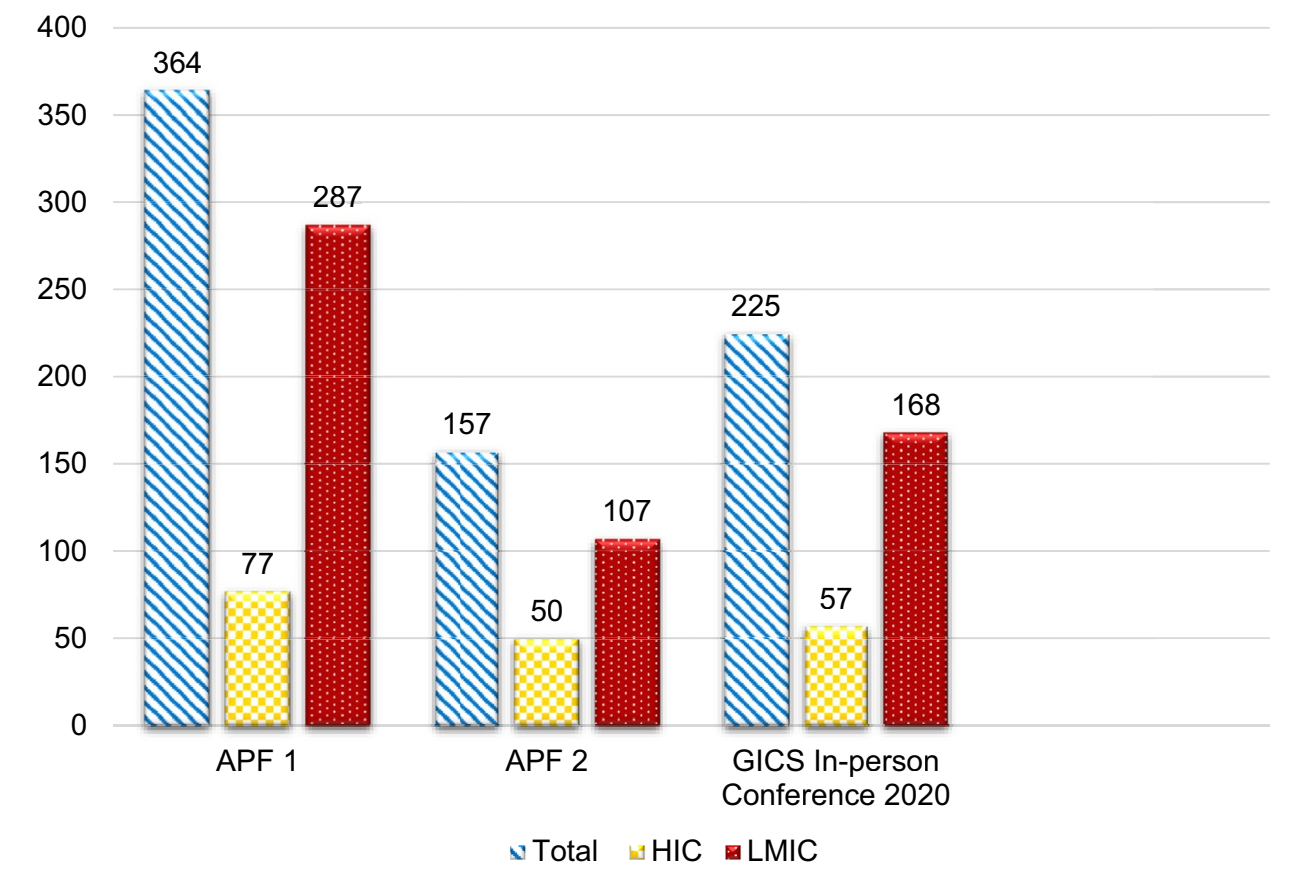

GICS: Global Initiative for Children's Surgery

APF: Action Planning Forum

HIC: High-income countries

LMIC: Low- and middle-income countries 
Table 2 Most frequently reported challenges at the Action Planning Forums

\begin{tabular}{lll}
\hline & Most frequently reported challenges & OReCS framework domain \\
\hline 1 & Cancelation of elective surgery & Service delivery \\
2 & Delayed access to care & Service delivery \\
Human resources and training \\
Infrastructure \\
\end{tabular}

mitigate the impact of the pandemic on children's surgery (Table 5).

\section{Educational resources}

Panelists described the need for three categories of up-to-date and readily accessible educational resources, including those for: senior surgeons, anesthesiologists and nurses; trainees and students; and the general public. Many of the panelists also noted the importance of utilizing online media and platforms for ongoing education and clinical training during the pandemic; however, there were mixed opinions about the utility of large versus small group sessions. In addition, panelists emphasized that engagement of local providers would require that the educational resources be translated and adapted to local context and needs.

\section{Guidelines}

All panelists unanimously agreed on the need for guidelines, checklists, and standard operating procedures (SOPs) for a wide variety of domains in children's surgery, which have been categorized into four main themes: safety of procedures, perioperative care, and healthcare professionals; adaptability to local needs; prioritization of essential, emergency, and elective surgical procedures; and accessibility of guidelines.

\section{Backlog}

Panelists expressed their concern about the denial of routine and urgent surgical care among children in their respective regions and made recommendations on possible ways to minimize the accumulating back$\log$. Bolstering the entire surgical workforce and providing support at all human resource levels-including anesthesia, technicians, and nurses-was cited as crucial for effectively dealing with the backlog of surgical cases. On the other hand, some panelists viewed increasing the surgical workforce as a major challenge and believed that alternative measures must be employed, including working extra hours and canceling leaves. Furthermore, a combination of funding, accurate data of patient volumes involved, telemedicine, engagement of local providers, and proper documentation of the backlog and unmet surgical need was considered important for effectively tackling the backlog.

\section{Research}

When asked about the potential role that GICS could play in supporting research efforts during the pandemic, many panelists recommended developing funded, multinational studies dedicated to children's surgery as well as systematic data collection on surgical outcomes among COVID-19-positive children. Additional research studies were proposed to explore the profile of pediatric surgical cases; safety of surgical procedures; financial impact of the pandemic; and impact of delayed surgical management secondary to the pandemic.

\section{Discussion}

\section{Online engagement}

To the best of our knowledge, this series of APFs was the first of its kind during the pandemic to engage providers from diverse regions and exchange expertise specifically within the field of children's surgery. Through these APFs, we were able to identify local needs and solutions dedicated to children's surgery in a time of crisis. The participation 
Table 3 The greatest challenges reported by panelists at the Action Planning Forums

\section{OReCS framework domains}

\section{Human resources and training}

Grief and trauma due to loss of colleagues and family members

\section{Shortage of skilled professionals}

Stressed health care workers (HCWs)

Interruption in hands-on training

\section{Infrastructure}

Diversion of resources (human, skills, infrastructure, supplies, financial) toward COVID-19 and critical care patients

Decrease in available beds and facilities dedicated to children's surgery

Unknown parent/caregiver COVID-19 status

\section{Service delivery}

Population level

a. Delayed access to appropriate surgical facilities

b. Management of suspected COVID-19 patients

Provider level

a. Cancelation of elective surgery

b. Lack of standard operating procedures (SOPs)/guidelines

\section{Equipment and supplies}

Lack of adequate PPE and COVID-19 testing

Improvisation to make PPE

\section{Quality improvement}

Delayed presentation

Increased length of hospital stay

\section{Advocacy and awareness}

Population level

a. Stigma and fear among patients and public
Panelists' thoughts/comments

In the Philippines, there were high numbers of COVID-19 cases, and tragedy struck close to home when Past-President of the Philippines Society of Pediatric Surgery and Chair of Pediatric Surgery at the Philippine Children's Medical Hospital, Dr Leondro Resurrection, died of COVID-19 complications early in the pandemic

Because many health care workers became infected with COVID-19, there were shortages of skilled nurses and physicians, and these caregivers were afraid; many nurses died from COVID-19 infection

There was a lack of psychological support for HCWs, who were often isolated from their families, denied life insurance, and in need of emotional support

Because fewer operations were being performed and clinic numbers were limited, the training of residents and fellows has been affected

Most resources in the country (are) directed toward ICU care and COVID-19 units

Because many of the central hospitals in the larger cities were converted to COVID-19 referral hospitals, the number of pediatric beds decreased drastically

Even when a pediatric patient tested negative, the COVID-19 status of his or her parents was not known, resulting in a constant risk to providers as these attendants changed throughout the hospitalization

There was little attention to children's surgery, with difficulties stemming from an inability to travel, closure of private hospitals, and patient rejection by some facilities

Many hospitals were dedicated to treating COVID-19 patients, but because it took $24-36 \mathrm{~h}$ for test results, it was unclear where to place the patients

In January the hospital cut elective surgery by $50 \%$, and only the primary surgeon and one assistant were present for each operation

After the first case of COVID-19, the national collegium and societies stopped elective surgery, allowing only emergent cases to be performed

There was a lack of consensus on which operations should be postponed and how long they should be delayed

There was insufficient PPE and unequal distribution, particularly at the 2nd level facilities

From the beginning, there was a lack of proper PPE; HCWs had to wear raincoats and other makeshift protection

In terms of pediatric orthopedic surgery, many patients were presenting late, with congenital anomalies presenting up to 2 months after birth, even from the same state

Length of stay for appendectomies increased because they presented late in their course, as did incarcerated hernias and torsed testes

There was a lack of clarity with regard to both public information and standard operating procedures, leading to confusion and stigma, with patients unwilling to give an accurate exposure history 
Table 3 (continued)

OReCS framework domains

b. Physical distancing and mask usage

Provider level

a. Panic among health care providers

b. Dearth of prior data on the novel SARS-CoV-2

\section{Governance and financing}

Imposition of lockdown

Rushed return to normal activity
Panelists' thoughts/comments

It was difficult to enforce 6-foot distancing or the wearing of masks, and it was difficult to educate the patients and their families

In Bangladesh, there was panic among the population and health care workers as they witnessed the deaths in Europe and North America, knowing that the resources were so much less in their country

HCWs feared infecting their family members

The primary challenge in Hong Kong was that very little was known about the virus at the beginning, when they were implementing policies

Rather than the virus itself, it was the lockdown that created the problems they saw, as patients were unable to travel for routine care

It was because of the secondary effects of poverty that the government decided to lift the lockdown on 31 May; the argument was that if people could not eat or feed their families, they would die from that rather than COVID-19 at the APFs surpassed that at the most recent in-person GICS Biennial Conference, held in January 2020 in Johannesburg, South Africa, where 225 delegates from 44 countries attended the 2-day proceedings (Fig. 1) [17]. Of note, some APF participants had never attended a GICS meeting before and were from countries not previously represented within our organization. Interestingly, the majority of participants at both the in-person conference $(74.7 \%)$ and the online APFs (APF 1: 78.8\% and APF 2: 68.2\%) were from LMICs. This is a reaffirmation of the mandate of GICS, and a testament of the ability of the APFs to reach a wide range of children's surgical care providers. A number of leading charitable organizations and philanthropists, including representatives from KidsOR, also attended these sessions. This was an important opportunity for these organizations to learn about the challenges and needs of the global children's surgery community directly from frontline providers in order for them to focus their resources and efforts in the right direction.

The strong participation noted at the online APFs, particularly from LMICs, can be attributed to multiple factors. First, even though GICS has always placed inclusion and equity at the core of its values and has provided travel funding for participants at each of its four global meetings (including for 150 of 225 participants at its most recent meeting), costs to attend in-person conferences are often exorbitant. The APFs offered an opportunity for engagement without travel costs. Second, because we recognized a sense of "webinar fatigue," we actively moved away from a didactic or lecture format, emphasizing that we sought practical solutions adapted to local needs based on our panelists' firsthand experiences. Third, most of our panelists were from LMICs and were at the forefront of tackling the pandemic in one way or another, which allowed attendees from low-resource settings to learn from the experiences and strategies shared by them. Finally, similar to findings from Patel et al.'s study [18], participants from our study may prefer the online format over in-person meetings, because it allows greater flexibility in terms of time and finances as participants also have the option to view recordings later at their convenience. This is especially true for participation from our LMIC colleagues. An online platform, conference, or session completely removes any financial or geographical barriers, promoting greater diversity and more equitable participation and contribution. Based on the response to the APFs and the widespread adoption of online forums during the travel restrictions imposed by the pandemic, it might be possible to increase participation in future GICS meetings by implementing virtual aspects to our biennial conference. While many GICS members find the in-person meetings important for hands-on training, fellowship, and networking, a hybrid approach might be considered to decrease expenses even as participation is maximized. Despite the benefits of online access, however, it is important to note that there was a significant drop in participation between the first and second forum, which can be attributed to a number of factors, including enhanced clinical and research responsibilities due to the COVID-19 pandemic. In addition, after attending the first forum, some people may have felt that the online forums had limited value, that there were better alternate resources available, or that they could just watch the recordings on YouTube later. Our mitigation strategy focused on sending emails to GICS members with a link to the APF recordings, as well as a summary of the APFs.

Multiple organizations and groups have utilized online webinars for the dissemination of knowledge, medical 
Table 4 The current and projected impact of the COVID-19 pandemic on global children's surgery

OReCS framework domains

\section{Human resources and training}

Education for trainees and students

Loss of healthcare providers

\section{Infrastructure}

Transition to telemedicine and remote clinics

\section{Service delivery}

Delayed/absent children's surgical care

Advanced disease presentation and higher rate of complications

Backlog of surgeries

Earlier access to tumor surgery for children on a waiting list

\section{Quality improvement}

Renewed emphasis on safety

\section{Governance and financing}

Loss of employment due to the lockdown/Economic impact
Panelists' thoughts/comments

While online training has gone up with the use of Zoom and other platforms, "hands on" training has dramatically decreased. Only the surgeons performing the operation are allowed into the operating rooms (ORs), affecting both residents and undergraduates

In the large population hotspots, health care workers were lost to COVID-19

Telemedicine allowed guidance of local doctors to care for some patients, and families were guided on how to adjust lengthening devices

No clinics have taken place since the lockdown began 2 months ago, with no plans yet to reopen, particularly in Lagos, where 50\% of COVID-19 cases have occurred

Most challenges were related to the inability of patients to come to the clinic for follow up or for their elective operations, with only $5 \%$ of last year's numbers presenting as new patients to the clinic

There is an expectation that many patients will present late or with complications from care rendered by less experienced doctors

There was limited OR time, for emergent and urgent cases only, leading to a huge backlog of patients

One benefit of the lockdown was that some children with tumors who were awaiting surgery were able to have their operations because there was suddenly space in the operating theaters for them

On a positive note, there has been a renewed emphasis on "safety first, safety second, and safety third," with everyone looking out for their colleagues' well-being

Another critical issue has been the loss of livelihood of daily workers due to the lockdown. Prior to COVID-19, Bangladesh had a poverty rate of $20.5 \%$; because of the economic disruption the poverty rate more than doubled to $43 \%$ education and training, and discussions of challenges and best practice guidelines during the current pandemic [18-21]. There is mounting evidence on ways that groups have mobilized quickly to form plans and act in the COVID19 era. Specifically, Tangcharoensathien and colleagues described the use of a 2-day global online consultation, organized by the WHO Information Network for Epidemics (EPI-WIN), to crowdsource ideas and effective strategies for tackling the COVID-19 infodemic and the spread of misinformation [22]. Similar to our study, the aforementioned study also included qualitative analysis of the ideas submitted to the online platform. In contrast to our study, this infodemic study engaged the public and incorporated their responses and feedback into shortlisting the top ideas. Furthermore, in a study by Salvador-Carulla et al., a rapid synthesis and translation process (RSTP) has been applied to a series of Pandemic-Mental Health International Network (Pan-MHIN) webinars to highlight experts' realworld experiences of effectively dealing with the pandemic
[23]. The RSTP is designed to allow for the rapid transfer of research-based and expert knowledge into action [24]. In a separate study, Attipoe-Dorcoo and colleagues shared their experience of hosting a webinar with over 300 mobile health providers to highlight challenges and best practices in coping with the COVID-19 pandemic and made recommendations on enhancing the application of mobile health programs [25]. Of note, in contrast to our study methodology, the authors employed an online survey in addition to a webinar to allow for a more targeted needs assessment of the mobile health programs. The GICS Nurses' Working Group similarly held a webinar to describe the specific challenges facing children's surgical nurses during the pandemic and to identify strategies for improving the quality of surgical nursing care. 
Table 5 Actions proposed by our panelists to effectively mitigate the impact of COVID-19 on children's surgery in the context of the Global Initiative for Children's Surgery (GICS) Optimal Resources for Children's Surgery (OReCS) document

Action item (OReCS framework domain) Panel's comments

Educational resources (human resources and training)

i. For all providers \& trainees

ii. For general public

iii. Online Media

iv. Large versus small groups

v. Adapted to local needs and resources

\section{Guidelines}

(Quality improvement)

\section{Safety}

i. Procedures

ii. Perioperative care

iii. Healthcare Professionals

Adaptability
i. Local needs

ii. Low- and Middle-income country (LMIC) representation

\section{Prioritization}

"First of all, about education, it is very important to have material not only for senior surgeons but also for junior surgeons, general surgery residents, fellows, and pediatricians. We had this experience yesterday. We did a webinar about the basic pediatric surgery for pediatricians, and we had more than 400 participants who thought this experience was very good."

"There is a dire need to provide public education via radio/TV shows to inform the public about the safety of visiting hospitals given all SOPs are followed."

"Once elective surgeries resume, GICS can always assist with the training of pediatric surgeons as well as the exchange of knowledge and skills through online media."

"I think both large webinars and smaller focus groups are very helpful in their own ways. We recently conducted a webinar on hypospadias, which was attended by 500 people. This allowed a greater reach and answering a lot of questions from the chat option."

"In Pakistan, there seems to be a preference for interactive sessions and small group discussions over webinars among surgeons and trainees. These small group sessions encourage easier communication of the day-to-day challenges faced during the pandemic."

"I also think that translation of fundamental material would be very important. Sometimes you think that everybody knows English, but I have some friends, colleagues, fellows who do not know how to speak English."

"One of the key topics requiring attention right now is the safety of conducting minimally invasive procedures, including laparoscopy and thoracoscopy in children."

"GICS could help with the development of standard safety protocols for pre-op, intra and post-op surgical care in children. This is especially important in Indonesia, where the number of pediatric surgeons is very low."

"We can have appropriate guidelines on appropriate PPE for different COVID-19 status, so what do you use for negative patients? What do you use for a suspected patient who has to go to the OR without a test? And, what do you use for the positive patient?"

"GICS could design and propose guidelines for patient preparation prior to surgery, role and importance of RT-PCR and other tests, prioritization of surgical cases and minimizing backlog, appropriate set up of operating rooms and anesthesia units, and safety of HCWs and other staff in the OR and Post-Anesthesia Care Unit (PACU)."

"About the guidelines, I think it's also important to have guidelines for institutions that don't have a lot of resources. I think that's not fair to have guidelines from only high-income countries. We also need something that is safe for low resource settings."

"Because each country and region have their own guidelines for PPE, testing, quarantine, and treatment, not all of these resources will be available in LMIC settings. However, people can see them and choose which ones apply. There must also be sufficient LMIC representation to know what is available and practical." 
Table 5 (continued)

\section{Action item (OReCS framework domain)}

i. Essential and emergency surgery

ii. Elective surgery

Accessibility

Backlog (human resources \& training; service delivery)

i. Strengthening the surgical workforce capacity

ii. Engaging local providers and telemedicine

\section{Research (Quality improvement)}

i. Profile of pediatric surgical cases

ii. Outcomes

iii. Safety of surgical procedures

iv. Financial burden

v. Delayed surgical management
Panel's comments

"There is also a need for guidelines on what procedures are considered essential and which are not from all subspecialties."

"In Africa, we are getting to the place of community spread as some countries are trying to open up, some are still having restriction measures involved, so how do people conduct themselves during this time when they have to do emergency surgery with the fact that there is community spread, where you are having patients coming in and you may not know their status, but you have to go to the operating room immediately."

"GICS could help with guidelines on the best practice of elective surgeries (e.g., hernia, circumcision, and specifically laparoscopic surgery), precautions for surgery of COVID-19 and non-COVID-19 patients."

"Another way that GICS could help would be to ensure that all relevant resources and recommendations are available in one place on the website. For example, it took me a very long time to find out that D-dimer tests for hypercoagulability can actually indicate the need for early anti-coagulation in symptomatic patients."

"Once we open and people are working, GICS can help with putting together surgical teams, who can travel from region to region offering surgery at surgical camps. This is especially in areas where we have very few providers and children have a very high burden of surgical diseases."

"It will also be important to involve local providers, even if they are general surgeons or pediatricians, to actually elicit findings when you are doing telemedicine. I find this very useful and I actually liaise with them all the time."

"Research should be done on the profile of pediatric surgical cases done during the pandemic in both high-income countries (HICs) and LMICs."

"In addition to gathering evidence to guide clinical practice, we also need to start looking at the community-based assessment of unmet need and the impact of COVID-19 on surgical outcomes."

"Research efforts should focus on long-term effects (including mental health and inflammatory conditions) of the pandemic on children."

"Research is needed on the safety of procedures and which alternatives can be used e.g., laparoscopy versus laparotomy, in order to keep meeting the demand of pediatric surgery."

"Additionally, financial stress can also be assessed by interviewing families, and I think this will be important when we approach donors to convey what financial stresses patients are suffering from."

"It would also be helpful to review patient records for the last 6-12 months to assess how many patients are missing essential visits that might affect them adversely."

"The information we can begin gathering now includes adverse outcomes, delay in starting essential treatments, such as casting clubfoot, delay in developmental dysplasia of the hip surgery, delay in removal or application of 8 plate."

\section{Challenges, impact, and proposed actions}

The panelists were queried about the greatest challenges facing them during the pandemic. The cancelation of elective surgery, delayed access to care for patients, fear among the public and patients, unavailability of appropriate PPE, diversion of resources toward COVID-19 care, and interruption of student and trainee education were most frequently cited. In addition, panelists highlighted that delayed surgery among children, especially for congenital anomalies, could translate into more advanced disease presentation and higher complication rates. These concerns are in line with previously published studies which demonstrate that congenital anomalies are responsible for 25.3-38.8 million disabilityadjusted life-years (DALYs) worldwide [26, 27], and of the estimated 21.6 million DALYs caused by cleft lip and palate, congenital heart disease, and neural tube defects in LMICs, 12.4 million DALYs are potentially avertable by surgical care [28]. Furthermore, a recent study by Roberton and colleagues estimates that disruptions in essential 
child health interventions due to the pandemic could result in 253,500-1,157,000 additional child deaths in LMICs over the next 6 months [11].

Recognizing that surgical problems often demand faceto-face interactions for diagnosis and treatment, our panelists described that the risk of viral transmission has shifted many patient-physician clinic interactions to telemedicine, although there are limitations in poorer locales [29]. In this context, Song and colleagues described the efficient and successful use of telemedicine in the Shandong province of China for patients, community residents, and medical staff for prevention and treatment guidance, training, and consultations [30]. Recently published work from children's surgical providers highlights their struggles in trying to maintain continuity of care despite lockdown, shortage of PPE, psychosocial distress experienced by patients and families, and overwhelmed hospital infrastructure [31]; this echoes the experiences shared by the APF panelists. In particular, in their study from Pakistan, Qazi et al. highlight their experiences of devising a priority list of pediatric surgical cases into three categories of elective, semi-elective, and emergency surgery, when elective surgery had to be canceled [31]. ${ }^{26}$ Similarly, Leva and colleagues describe the drastic modifications that have been made to their pediatric surgical services due to the COVID-19 pandemic [32]. Specifically, the number of pediatric beds decreased from 28 to 12,9 out of 15 pediatric nurses were moved to other areas within the hospital, increased rates of complicated acute appendicitis were observed, and only 4 children requiring emergency surgery that could not be delayed had been treated by April 2020 [32].

Panelists noted the interruptions in hands-on clinical training for nursing and medical students, residents, and fellows. Similar reports have been described by other educators and clinicians from all over the world [31, 33-35]. Multiple platforms, including online webinars and training, surgical simulation sessions, and telemedicine apps have been used by institutions globally [31, 36, 37]. It is important to note, however, that many institutions and providers in low-resource settings may not have access to paid versions of online software or surgical simulation techniques. Therefore, as mentioned by some of our panelists, locally applicable solutions must be employed. In addition, sharing of information about free and easily accessible resources is helpful. McKechnie et al. have provided a comprehensive list of computer- and phone-based resources for surgical education, many of which are freely available [36]. Moreover, our panelists emphasized the need to conduct research on children's surgery in the COVID-19 era. COVIDSurg is an international, multi-center prospective cohort study which aims to assess outcomes among COVID-19-positive patients undergoing surgery $[38,39]$. COVIDSurg includes patients from all age groups, and therefore does not have a specific focus on the pediatric age group, which has inherently different dynamics. Multiple international organizations have partnered to assess the impact of the pandemic on pediatric cancer services and their outcomes as part of an international multi-center study called COVIDPaedsCancer [40]. This study focuses specifically on childhood cancer including surgical management of cancers. In light of the dire need to ensure systematic data collection for surgical outcomes among COVID-19-positive children as well as the current and projected impact of the pandemic on children's surgery, conducting an international, multi-center study may be beneficial.

For the past few months, GICS has been working actively to implement many of the proposed actions from the APFs. For guidelines, the GICS leadership realized that this task was heavily dependent on resources and adding another set of guidelines to the existing pool of a substantial number of guidelines and recommendations would not be as fruitful as focusing efforts on education, research, and minimizing backlog. With regards to minimizing backlog, reports on the resumption of elective surgery are emerging from different parts of the world. Specifically, Tafoya and Tumber share their experience of reopening for pediatric surgery cases in mid-April 2020 by assessing local COVID-19-infected case prevalence, using reusable powered air-purifying respirators (PAPRs) for the majority of cases, shifting to an urgencybased schedule, and developing a COVID-19 Perioperative Testing Decision Tree [41]. Okonkwo et al. showed that after resuming elective surgery in May 2020, children and young people undergoing elective surgery had outcomes similar to pre-COVID era, given that appropriate household selfisolation, testing, and screening were done. [42] The GICS Education committee is working on designing education modules, cases, virtual grand rounds, and small group sessions to address the disruption in clinical training. GICS has also initiated a global survey to assess the impact of the pandemic on children's surgical care.

\section{Role of the Optimal Resources for Children's Surgical Care (OReCS) document}

The OReCS, a comprehensive document developed by multiple subspecialty providers in GICS, is published and available on the GICS website $[16,43]$. OReCS provides an indepth description of the resources and infrastructure required to ensure the provision of safe surgical and anesthesia care for children at all facility levels in the healthcare system. It does so by highlighting four key types of resources: human resources, skills, infrastructure, and equipment/supplies. OReCS has been divided into nine framework domains, namely: types of healthcare facilities, human resources and training, tasks and responsibilities, infrastructure, service delivery, equipment and supplies, quality improvement, 
advocacy and awareness, and governance and financing. By deconstructing children's surgical care into readily comprehensible components, the OReCS framework provides an easily applicable guide during routine practice as well as during times of crisis. The strength and applicability of the OReCS document has been highlighted during our analysis, allowing challenges, impact, and proposed actions to be defined in this context.

\section{Strengths and limitations}

Our study is novel and important, because it is the first to do a targeted assessment of the collateral damage to the global children's surgical community as well as obtain a consensus on the interventions best suited to mitigate the impact of the pandemic on children's surgery. Furthermore, we have employed an objective and transparent method of analyzing and presenting findings from our action planning forums. Research groups can replicate this methodology in the future.

There are several limitations to consider in this study. First, we were not able to address all questions which were asked by participants in the chat box. Second, following the APFs, the GICS leadership and membership identified ways in which to provide support to the panelists and other providers tackling the pandemic; however, the proposed actions are still in the planning phases, so it is too soon to assess the mitigating impact of the APFs. Third, some of the proposed actions or interventions, including provision of PPE or funding for testing kits, are beyond the funding capabilities of GICS. Nonetheless, it is important to identify these critical shortages to inform donors and policy makers.

\section{Conclusion}

The COVID-19 pandemic has far-reaching consequences for surgical systems globally, with children's surgery at risk of being disproportionately affected. Diversion of resources away from children's surgery threatens to exacerbate further the existing unmet surgical need among neonates, children, and adolescents. Interruption in clinical training and research activities also have the potential to hinder progress in the field of children's surgery. Even though it is premature to suggest how effective the APFs will be in mitigating the impact of the pandemic on children's surgery, identification of key challenges and locally adaptable interventions is an important first step. Currently, the GICS leadership and membership are working with their partner organizations to plan and implement many of the proposed actions, and a regular review of the effectiveness of these APFs and proposed interventions has been planned.
Author contributions AA, LS, DO, and MWB contributed to the study conception and design. Data collection and analysis were performed by AA and MWB. The first draft of the manuscript was written by AA, reviewing and editing were performed by AA, LS, DO, and MWB, and all authors commented on previous versions of the manuscript. All authors read and approved the final manuscript.

Funding No funding was received to assist with the preparation of this manuscript.

\section{Compliance with ethical standards}

Conflicts of interest The authors have no relevant financial or nonfinancial interests to disclose.

Ethics approval This study was granted institutional review board (IRB) exemption from the IRD IRB at the Indus Hospital, Karachi, Pakistan (IRB number: IRD_IRB_2020_12_005).

Consent to participate Informed consent was obtained from all panelists prior to the online action planning forums.

Consent to publish All panelists and authors have consented to the submission of the manuscript to the journal.

\section{References}

1. World Health Organization (2020) Coronavirus disease (COVID19) Situation Report-164 [Internet]. Available from: https://www. who.int/docs/default-source/coronaviruse/situation-reports/20200 702-covid-19-sitrep-164.pdf?sfvrsn=ac074f58_2. Cited 11 Sep 2020

2. Organization WH. Coronavirus disease (COVID-19) (2020) Situation Report - 198 [Internet]. Available from: https://www.who. int/docs/default-source/coronaviruse/situation-reports/20200805covid-19-sitrep-198.pdf?sfvrsn=f99d1754_2. Cited 20 Oct 2020

3. Kim L, Whitaker M, O'Halloran A, Kambhampati A, Chai SJ, Reingold A et al (2020) Hospitalization Rates and Characteristics of Children Aged \&lt; 18 Years Hospitalized with LaboratoryConfirmed COVID-19 - COVID-NET, 14 States, March 1-July 25, 2020. MMWR Morbidity and Mortality Weekly Report [Internet]. 2020 Aug 14 [cited 2020 Sep 11];69(32):1081-8. Available from: http://www.cdc.gov/mmwr/volumes/69/wr/mm6932e3. htm?s_cid=mm6932e3_w

4. Docherty AB, Harrison EM, Green CA, Hardwick HE, Pius R, Norman L et al (2020) Features of 16,749 hospitalised UK patients with COVID-19 using the ISARIC WHO Clinical Characterisation Protocol. medRxiv [Internet]. 2020 Apr 28 [cited 2020 Sep 11];10:2020.04.23.20076042. Available from: https:// www.medrxiv.org/content/https://doi.org/10.1101/2020.04.23. 20076042v1

5. Wu Z, McGoogan JM (2020) Characteristics of and Important Lessons from the Coronavirus Disease 2019 (COVID-19) Outbreak in China: Summary of a Report of 72314 Cases from the Chinese Center for Disease Control and Prevention [Internet]. Vol. 323, JAMA - Journal of the American Medical Association. American Medical Association; 2020 [cited 2020 Sep 11]. p. 1239-1242. Available from: https://jamanetwork.com/

6. Dong Y, Dong Y, Mo X, Hu Y, Qi X, Jiang F, et al. Epidemiology of COVID-19 among children in China. Pediatrics. 2020;145(6).

7. Ahmed M, Advani S, Moreira A, Zoretic S, Martinez J, Chorath $\mathrm{K}$, et al. Multisystem inflammatory syndrome in children: A 
systematic review. EClinicalMedicine [Internet]. 2020 Sep 1 [cited 2020 Oct 20];26. https://doi.org/10.1016/j.eclinm.2020. 100527

8. Abrams JY, Godfred-Cato SE, Oster ME, Chow EJ, Koumans EH, Bryant B et al (2020) Multisystem inflammatory syndrome in children associated with severe acute respiratory syndrome coronavirus 2: a systematic review. Journal of Pediatrics [Internet]. 2020 [cited 2020 Oct 20]; Available from: /pmc/articles/ PMC7403869/?report=abstract

9. Verdoni L, Mazza A, Gervasoni A, Martelli L, Ruggeri M, Ciuffreda $M$ et al (2020) An outbreak of severe Kawasaki-like disease at the Italian epicentre of the SARS-CoV-2 epidemic: an observational cohort study. The Lancet [Internet]. 2020;395(10239):17718. Available from: http://dx.doi.org/https://doi.org/10.1016/ S0140-6736(20)31103-X

10. Khan KS, Ullah I (2020) SARS-CoV-2 causes Kawasaki-like disease in children: Cases reported in Pakistan. J Med Virol. 2020;2-3.

11. Roberton T, Carter ED, Chou VB, Stegmuller AR, Jackson BD, Tam Y et al (2020) Early estimates of the indirect effects of the COVID-19 pandemic on maternal and child mortality in low-income and middle-income countries: a modelling study. The Lancet Global Health [Internet]. 2020 Jul 1 [cited 2020 Sep 15];8(7):e901-8. Available from: www.thelancet.com/lancetgh

12. Davenport M, Pakarinen MP, Tam P, Laje P, Holcomb GW (2020) From the editors: the COVID-19 crisis and its implications for pediatric surgeons. J Pediatr Surg 55(5):785-788

13. Mazingi D, Ihediwa G, Ford K, Ademuyiwa AO, Lakhoo K (2020) Mitigating the impact of COVID-19 on children's surgery in Africa. BMJ Glob Health 5(6):1-5

14. Home - Global Initiative for Children's Surgery [Internet]. [cited 2020 Oct 20]. Available from: https://www.globalchildrenssurge ry.org/

15. Homepage: Global Surgery Organization - Lifebox [Internet]. [cited 2020 Oct 20]. Available from: https://www.lifebox.org/

16. Grabski D, Ameh E, Ozgediz D, Oldham K, Abantanga FA, Abdelmalak M et al (2020) Optimal Resources for Children's Surgical Care: Executive Summary. World Journal of Surgery [Internet]. 2019 Apr 15 [cited 2020 Aug 22];43(4):978-80. Available from: https://pubmed.ncbi.nlm.nih.gov/30725368/

17. Vervoort D (2020) Saving the future through the art of the knife [Internet]. Global Health NOW. 2020 [cited 2020 Sep 15]. Available from: https://www.globalhealthnow.org/2020-01/savingfuture-through-art-knife

18. Patel NM, Khajuria A, Khajuria A (2020) Utility of a webinar to educate trainees on UK core surgical training CST ) selection - a cross sectional study and future implications amidst the COVID19 pandemic

19. Ficarra V, Novara G, Abrate A, Bartoletti R, Crestani A, De Nunzio $C$ et al (2020) Urology practice during the COVID-19 pandemic. Minerva urologica e nefrologica. Ital J Urol Nephrol. 72(3):369-75

20. Ali SR, Dobbs TD, Whitaker IS (2020) Webinars in plastic and reconstructive surgery training - a review of the current landscape during the COVID-19 pandemic [Internet]. Vol. 73, Journal of Plastic, Reconstructive and Aesthetic Surgery. Churchill Livingstone; 2020 [cited 2020 Sep 15]. p. 1357-404. Available from: https://www.ncbi.nlm.nih.gov/pmc/articles/PMC7243775/

21. Liu RB, Tayal VS, Panebianco NL, Tung-Chen Y, Nagdev A, Shah S, et al (2020) Ultrasound on the Frontlines of COVID19: Report From an International Webinar [Internet]. Vol. 27, Academic Emergency Medicine. Blackwell Publishing Inc. [cited 2020 Sep 15]. p. 523-6. Available from: /pmc/articles/ PMC7267389/?report=abstract

22. Tangcharoensathien V, Calleja N, Nguyen T, Purnat T, D’Agostino M, Garcia-Saiso S et al (2020) Framework for
Managing the COVID-19 Infodemic: Methods and Results of an Online, Crowdsourced WHO Technical Consultation. Journal of medical Internet research [Internet]. 2020 Jun 1 [cited 2020 Sep 14];22(6):e19659. Available from: /pmc/articles/ PMC7332158/?report=abstract

23. Salvador-Carulla L, Rosenberg S, Mendoza J, Tabatabaei-Jafari H (2020) Rapid response to crisis: Health System Lessons from the active period of COVID-19: A Framework for Rapid Response. Health policy and technology [Internet]. 2020 Aug 27 [cited 2020 Sep 14];446. Available from: http://www.ncbi.nlm.nih.gov/pub$\mathrm{med} / 32874862$

24. Thigpen S, Puddy RW, Singer HH, Hall DM (2012) Moving knowledge into action: developing the rapid synthesis and translation process within the interactive systems framework. American Journal of Community Psychology [Internet]. 50(3-4):285-94. Available from: /pmc/articles/PMC4739647/?report=abstract

25. Attipoe-Dorcoo S, Delgado R, Gupta A, Bennet J, Oriol NE, Jain SH (2020) Mobile health clinic model in the COVID-19 pandemic: Lessons learned and opportunities for policy changes and innovation [Internet]. Vol. 19, International Journal for Equity in Health. BioMed Central Ltd.; 2020 [cited 2020 Sep 15]. Available from: /pmc/articles/PMC7236869/?report=abstract

26. Colin Mathers TB and DMF (2008) The global burden of disease 2004 update. Geneva: World Health Organization; 2004;146.

27. Murray CJL, Vos T, Lozano R, Naghavi M, Flaxman AD, Michaud C, et al (2012) Disability-adjusted life years (DALYs) for 291 diseases and injuries in 21 regions, 1990-2010: a systematic analysis for the Global Burden of Disease Study 2010. The Lancet [Internet]. 2012 Dec 1 [cited 2020 Sep 15];380(9859):2197-223. Available from: https://pubmed.ncbi.nlm.nih.gov/23245608/

28. Higashi H, Barendregt JJ, Kassebaum NJ, Weiser TG, Bickler SW, Vos T (2015) The burden of selected congenital anomalies amenable to surgery in low and middle-income regions: Cleft lip and palate, congenital heart anomalies and neural tube defects. Arch Dis Childhood [Internet]. 2015 Mar 1 [cited 2020 Sep 15];100(3):233-8. Available from: https://pubmed.ncbi.nlm.nih. gov/25260520/

29. Bhaskar S, Bradley S, Chattu VK, Adisesh A, Nurtazina A, Kyrykbayeva S et al (2020) Telemedicine As The New Outpatient Clinic Gone Digital: Position Paper From The Pandemic Health System REsilience PROGRAM (REPROGRAM $\dagger$ ) International Consortium (Part 2). Frontiers in Public Health [Internet]. 8:410. Available from: www.frontiersin.org

30. Song X, Liu X, Wang C (2020) The role of telemedicine during the COVID-19 epidemic in China - Experience from Shandong province [Internet]. Vol. 24, Critical Care. BioMed Central; 2020 [cited 2020 Sep 15]. p. 178. Available from: https:// ccforum.biomedcentral.com/articles/https://doi.org/10.1186/ s13054-020-02884-9

31. Qazi SH, Saleem A, Pirzada AN, Hamid LR, Dogar SA, Das JK (2020) Challenges to delivering pediatric surgery services in the midst of COVID 19 crisis: experience from a tertiary care hospital of Pakistan. Pediatric Surgery International [Internet]. Available from: https://doi.org/https://doi.org/10.1007/s00383-020-04721-0

32. Leva E, Morandi A, Sartori A, Macchini F, Berrettini A, Manzoni G (2020) Correspondence from Northern Italy about our experience with COVID-19 [Internet]. Vol. 55, Journal of Pediatric Surgery. W.B. Saunders; 2020 [cited 2020 Sep 15]. p. 985-6. Available from: https://doi.org/https://doi.org/10.1016/j.jpedsurg.2020. 03.028

33. Alyami AH, Alyami AA, AlMaeen BN (2020) Impact of COVID19 on orthopedic surgery: Experience from Saudi Arabia. Annals of Medicine and Surgery [Internet]. 2020 Aug 1 [cited 2020 Sep 16];56:61-3. Available from: https://www.ncbi.nlm.nih.gov/pmc/ articles/PMC7286826/ 
34. Osama M, Zaheer F, Saeed H, Anees K, Jawed Q, Syed SH et al (2020) Impact of COVID-19 on surgical residency programs in Pakistan; A residents' perspective. Do programs need formal restructuring to adjust with the "new normal"? A cross-sectional survey study. International Journal of Surgery [Internet]. $2020 \mathrm{Jul}$ 1 [cited 2020 Sep 16];79:252-6. Available from: /pmc/articles/ PMC7280820/?report=abstract

35. Zheng J, Hundeyin M, He K, Sachs T, Hess DT, Whang E et al (2020) General surgery chief residents' perspective on surgical education during the coronavirus disease 2019 (COVID-19) pandemic. Surgery (United States) [Internet]. 2020 Aug 1 [cited 2020 Sep 16];168(2):222-5. Available from: https://pubmed.ncbi.nlm. nih.gov/32600881/

36. McKechnie T, Levin M, Zhou K, Freedman B, Palter VN, Grantcharov TP (2020) Virtual Surgical Training During COVID19: Operating Room Simulation Platforms Accessible From Home. Annals of surgery [Internet]. 2020 Aug 1 [cited 2020 Sep 16];272(2):e153-4. Available from: https://www.ncbi.nlm.nih. gov/pmc/articles/PMC7268842/

37. Chandratre S (2020) Medical students and COVID-19: challenges and supportive strategies. Journal of Medical Education and Curricular Development [Internet]. 2020 Jan [cited 2020 Sep 16];7:238212052093505. Available from: /pmc/articles/ PMC7315659/?report=abstract

38. Nepogodiev D, Bhangu A, Glasbey JC, Li E, Omar OM, Simoes JF et al (2020) Mortality and pulmonary complications in patients undergoing surgery with perioperative SARS-CoV-2 infection: an international cohort study. The Lancet [Internet]. $2020 \mathrm{Jul} 4$ [cited 2020 Sep 16];396(10243):27-38
39. CovidSurg - Globalsurg [Internet]. [cited 2020 Sep 16]. Available from: https://globalsurg.org/covidsurg/

40. Global Children's NCDs Newsletter \#3 [Internet]. [cited 2020 Sep 16]. Available from: https://mailchi.mp/d36e35d79b57/excitingbeginnings-global-childrens-ncds-newsletter-2784946? $=8 \mathrm{eb} 56$ $\mathrm{c} 4 \mathrm{a} 2 \mathrm{f}$

41. Tafoya S, Tumber S (2020) Pediatric anesthesia during the coronavirus disease epidemic - One pediatric surgical hospital's rapid transition back to care. Pediatric Anesthesia [Internet]. 2020 Nov 3 [cited 2021 Mar 14];30(11):1275-7. Available from: https:// onlinelibrary.wiley.com/doi/https://doi.org/10.1111/pan.14000

42. Okonkwo INC, Howie A, Parry C, Shelton CL, Cobley S, Craig R et al (2020) The safety of paediatric surgery between COVID-19 surges: an observational study. Anaesthesia [Internet]. $2020 \mathrm{Dec}$ 1 [cited 2021 Mar 14];75(12):1605-13. Available from: /pmc/ articles/PMC7537528/?report=abstract

43. Goodman LF, St-Louis E, Yousef Y, Cheung M, Ure B, Ozgediz D et al (2018) The Global Initiative for Children's Surgery: Optimal Resources for Improving Care. Euro2018pean Journal of Pediatric Surgery [Internet]. 2018 Feb 1 [cited 2020 Sep 16];28(1):51-9. Available from: https://pubmed.ncbi.nlm.nih.gov/28806850/

Publisher's Note Springer Nature remains neutral with regard to jurisdictional claims in published maps and institutional affiliations.

\section{Authors and Affiliations}

\section{Alizeh Abbas ${ }^{1}$ - Lubna Samad ${ }^{1}$. Doruk Ozgediz ${ }^{2}$. Adesoji Ademuyiwa ${ }^{3} \cdot$ Emmanuel A. Ameh $^{4} \cdot$ Tahmina Banu $^{5}$. Fabio Botelho ${ }^{6} \cdot$ Beda Espineda $^{7} \cdot$ Zipporah Gathuya $^{8} \cdot$ Kokila Lakhoo $^{9} \cdot$ Lawal-Aiyedun Olubunmi $^{10}$. Vrisha Madhuri ${ }^{11}$. Leecarlo Millano ${ }^{12}$. Susane Nabulindo ${ }^{13}$. Sameh Shehata ${ }^{14} \cdot$ Kenneth Wong $^{15}$. Marilyn W. Butler ${ }^{16}$. On behalf of the Global Initiative for Children's Surgery}

1 Center for Essential Surgical and Acute Care, Global Health Directorate, Indus Health Network, Woodcraft Building, Plot 3 and 3A, Sector 47, Korangi Creek Road, Karachi, Pakistan

2 University of California, San Francisco, San Francisco, CA, USA

3 Paediatric Surgery Unit, Department of Surgery, Faculty of Clinical Sciences, College of Medicine, University of Lagos, Lagos, Nigeria

4 Division of Paediatric Surgery, Department of Surgery, National Hospital, Abuja, Nigeria

5 Chittagong Research Institute for Children Surgery, Chittagong, Bangladesh

6 Hospital das Clinicas da Universidade Federal de Minas Gerais, Belo Horizonte, Brazil

7 Philippines Children's Medical Center, Quezon City, The Philippines

8 The Nairobi Hospital, Nairobi, Kenya
$9 \quad$ Nuffield Department of Surgical Sciences, University of Oxford, Oxford, UK

10 Spina Bifida and Hydrocephalus Care Foundation, Abuja, Nigeria

11 Department of Paediatric Orthopaedics, Christian Medical College and Hospital, Vellore, India

12 Pediatric Surgery Division, Tarakan General Hospital, Jakarta, Indonesia

13 Department of Anaesthesia, University of Nairobi, Nairobi, Kenya

14 Department of Pediatric Surgery, University of Alexandria, Alexandria, Egypt

15 Department of Surgery, The University of Hong Kong, Hong Kong, Hong Kong

16 Division of Pediatric Surgery, Oregon Health and Science University, 501 N. Graham Street, Suite 300, Portland, OR 97227, USA 